\title{
End TB with precision treatment!
}

\author{
To the Editor:
}

We read with interest the recent Perspective paper by LöNnRoth et al. [1] describing a framework for tuberculosis (TB) elimination. In our opinion, monitoring drug exposure by measuring blood concentrations over time (therapeutic drug monitoring (TDM)) may be helpful in contributing to TB elimination in low- and medium-burden setting.

Treatment with the four first-line drugs (isoniazid, rifampicin, pyrazinamide and ethambutol), though usually successful, is increasingly challenged by the emergence of drug resistance, toxicity, relapse and nonresponse.

For a drug to be effective, both its action at the site of the disease process (pharmacodynamics) and the drug concentration over time (pharmacokinetics) are equally important. For most TB drugs, the area under the curve (AUC) is the most important pharmacokinetics parameter [2]. Drug exposure may be influenced by several different factors, such as concomitant food intake, comorbidities, co-medication and intra-individual differences in pharmacokinetics. Patients prone to low drug exposures are those with malabsorption and gastro-intestinal disorders, patients who have drug-drug interactions, and patients with diabetes mellitus or HIV co-infection. More importantly, pharmacokinetic variability is the driver of drug resistance [2]. In these individuals, there is a rationale for TDM.

It is not only the above-mentioned external factors that are important; the first-line TB drugs themselves are also problematic. The variability of rifampin plasma drug concentrations over time are influenced by its auto-inducing capacity, lowering rifampin exposure by $40 \%$ after 40 days when the induction is maximised [3]. The variability of isoniazid exposure is further influenced by $N$-acetyltransferase 2 (NAT2), which metabolises it to non-hepatotoxic metabolites. Generally, slow acetylators exhibit higher isoniazid plasma concentrations than rapid acetylators [4]. In a randomised clinical trial, isoniazid dosing adjusted for the NAT2 genotype resulted in reduced toxicity and decreased treatment failure [5]. Clearance of isoniazid, rifampin and pyrazinamide is a metabolic process handled by liver enzymes. Altered or impaired hepatic function further complicates dosing of these drugs. As it is rather difficult to quantify the metabolising function of the liver, TDM is the only way to ascertain adequate dosing. Three of the first-line TB drugs (isoniazid, rifampin and pyrazinamide) are potentially hepatotoxic [6] and more so if plasma concentrations increase [7]. TDM may therefore also prevent toxicity if performed timely.

Poor treatment outcome has been associated with low TB drug exposure, with an almost nine-fold increase in treatment failure in patients with low drug exposure [8]. In order to achieve a favourable outcome, the AUC of pyrazinamide should be $>363$, rifampin should be $>13$ and isoniazid should be $>52$ $\mathrm{mg} \cdot \mathrm{h} \cdot \mathrm{L}^{-1}$. Low maximum concentration to minimum inhibitory concentration ratios preceded acquired drug resistance [8]. Clearly, these data call for TDM.

For TB treatment, the time has come to move away from a one-size-fits-all approach; we need individualised approaches with considerably more precision. In figure 1, a strategy to use TDM on top of regular care is shown. We realise that this statement is more of a hypothesis or viewpoint than a scientifically proven fact. Indeed, scientific evidence is limited at this point in time. A randomised clinical trial should therefore explore the potential benefit of TDM in TB treatment. Standard treatment should be compared with TDM-guided dosing in combination with minimum inhibitory concentration testing. The study should be powered to detect both clinically and epidemiologically meaningful differences in relapse rate, acquired drug resistance or toxicity. TDM implementation in TB treatment need not necessarily increase cost. Even in high TB-burdened, resource-poor countries, TDM may prove cost-effective. Local healthcare workers could be taught to take patient samples. Dried blood spot sampling has considerable logistic and financial advantages, involving easier sampling, storage and transportation, making TDM an attainable goal in remote, poorly resourced areas [9].

Once proven cost-effective, it seems logical to team up with the Global Laboratory Initiative (GLI) of the World Health Organization (WHO), whose aim is to strengthen and expand TB laboratory capacity [10].

For several TB drugs, physicians have used TDM in TB treatment if toxicity was suspected or if patients responded unfavourably. Indeed, TDM is "ready for prime time". Instead of working in the dark, with a gunshot approach, clinicians should switch on the light provided by TDM and support their clinical decisions with current technologies to hit their target with much more precision. 


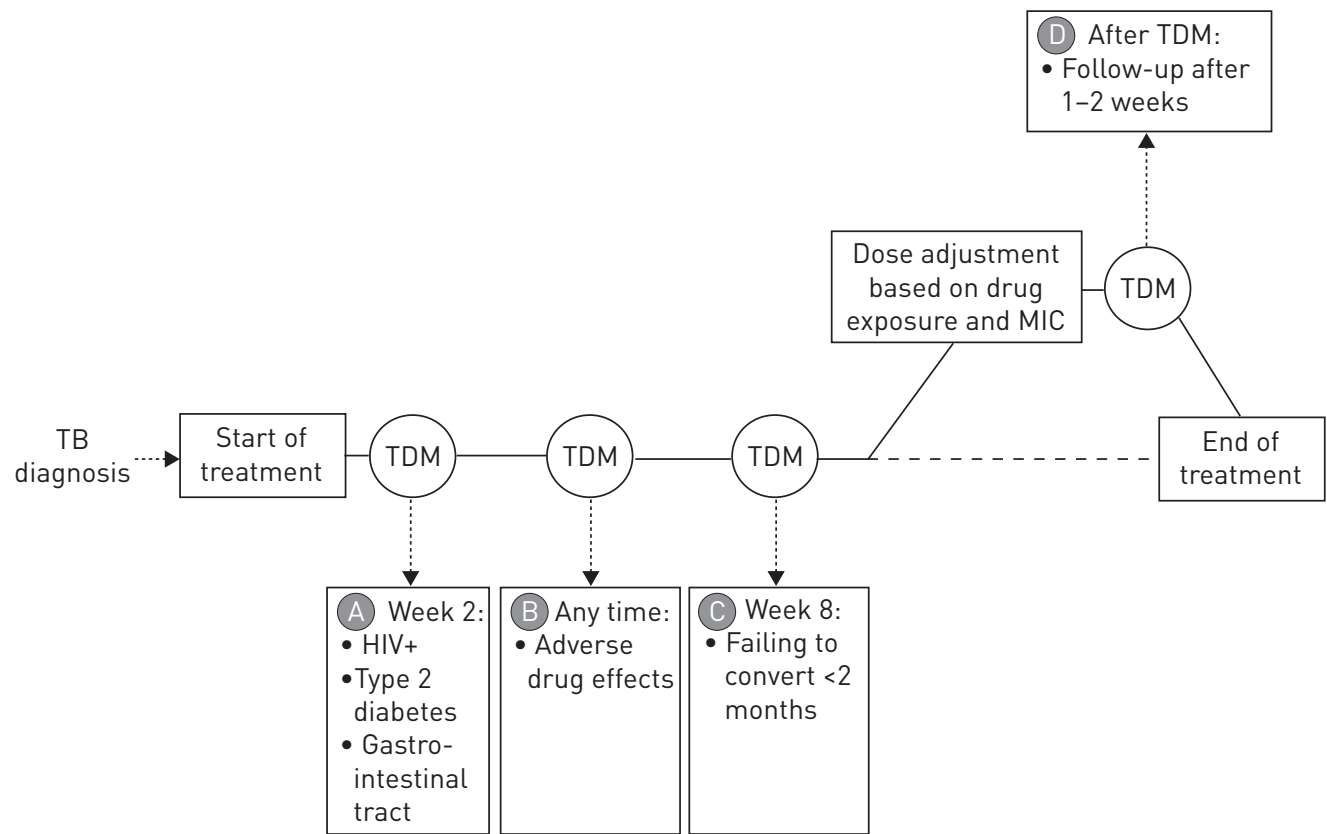

FIGURE 1 Therapeutic drug monitoring (TDM) in a programmatic setting. During treatment, TDM should be initiated by a) patient characteristics potentially resulting reduced drug exposure, b) adverse drug effects that may happen at any point in time during treatment, or c) patients failing to convert their sputum (microscopy and/or culture) within 2 months. d) Dose adjustment should be followed by verification if drug exposure is adequate. TB: tuberculosis; MIC: minimal inhibitory concentration.

$@$ ERSpublications

TB precision treatment, including optimised drug exposure in relation to drug susceptibility testing, may help end TB http://ow.ly/Utu2u

Evelien P.M. van der Burgt ${ }^{1}$, Marieke G.G. Sturkenboom ${ }^{1}$, Mathieu S. Bolhuis ${ }^{1}$, Onno W. Akkerman ${ }^{2,3}$, Jos G.W. Kosterink ${ }^{1}$, Wiel C.M. de Lange ${ }^{2,3}$, Frank G.J. Cobelens ${ }^{4,5}$, Tjip S. van der Werf ${ }^{3,6}$ and Jan-Willem C. Alffenaar ${ }^{1}$ ${ }^{1}$ University of Groningen, University Medical Center Groningen, Dept of Clinical Pharmacy and Pharmacology, Groningen, The Netherlands. ${ }^{2}$ University of Groningen, University Medical Center Groningen, Dept of Pulmonary Diseases and Tuberculosis, Groningen, The Netherlands. ${ }^{3}$ University of Groningen, University Medical Center Groningen, Tuberculosis Center Beatrixoord, Haren, The Netherlands. ${ }^{4} \mathrm{KNCV}$ Tuberculosis Foundation, The Hague, The Netherlands. ${ }^{5}$ Amsterdam Institute for Global Health and Development, Amsterdam, The Netherlands. ${ }^{6}$ University of Groningen, University Medical Center Groningen, Dept of Internal Medicine, Groningen, The Netherlands.

Correspondence: Dept of Clinical Pharmacy and Pharmacology, University Medical Center Groningen, PO Box 30.001, 9700 RB Groningen, The Netherlands. E-mail: j.w.c.alffenaar@umcg.nl

Received: July 202015 | Accepted after revision: Aug 292015

Conflict of interest: None declared.

\section{References}

1 Lönnroth K, Migliori GB, Abubakar I, et al. Towards tuberculosis elimination: an action framework for low-incidence countries. Eur Respir J 2015; 45: 928-952.

2 Srivastava S, Pasipanodya JG, Meek C, et al. Multidrug-resistant tuberculosis not due to noncompliance but to between-patient pharmacokinetic variability. J Infect Dis 2011; 204: 1951-1959.

3 Smythe W, Khandelwal A, Merle C, et al. A semimechanistic pharmacokinetic-enzyme turnover model for rifampin autoinduction in adult tuberculosis patients. Antimicrob Agents Chemother 2012; 56: 2091-2098.

4 Conte JE Jr, Golden JA, Mcquitty M, et al. Effects of gender, AIDS, and acetylator status on intrapulmonary concentrations of isoniazid. Antimicrob Agents Chemother 2002; 46: 2358-2364.

5 Azuma J, Ohno M, Kubota R, et al. NAT2 genotype guided regimen reduces isoniazid-induced liver injury and early treatment failure in the 6-month four-drug standard treatment of tuberculosis: a randomized controlled trial for pharmacogenetics-based therapy. Eur J Clin Pharmacol 2013; 69: 1091-1101.

6 Tostmann A, Boeree MJ, Aarnoutse RE, et al. Antituberculosis drug-induced hepatotoxicity: concise up-to-date review. J Gastroenterol Hepatol 2008; 23: 192-202.

7 Satyaraddi A, Velpandian T, Sharma SK, et al. Correlation of plasma anti-tuberculosis drug levels with subsequent development of hepatotoxicity. Int J Tuberc Lung Dis 2014; 18: 188-195.

8 Pasipanodya JG, McIlleron H, Burger A, et al. Serum drug concentrations predictive of pulmonary tuberculosis outcomes. J Infect Dis 2013; 208: 1464-1473. 
9 Vu DH, Alffenaar JW, Edelbroek PM, et al. Dried blood spots: a new tool for tuberculosis treatment optimization. Curr Pharm Des 2011; 17: 2931-2939.

10 Esposito S, Codecasa LR, Centis R. The role of therapeutic drug monitoring in individualised drug dosage and exposure measurement in tuberculosis and HIV co-infection. Eur Respir J 2015; 45: 571-574.

\section{B-blockers in pulmonary arterial hypertension: generation might matter}

To the Editor:

We read with great interest the article from BANDYOPADHYAY et al. [1] regarding the outcomes of $\beta$-blockers use in pulmonary arterial hypertension $(\mathrm{PAH})$. This study adds another piece in the very controversial debate opposing pro- and anti-supporters of $\beta$-blockers use in PAH. In this study, patients had idiopathic PAH (IPAH) or connective tissue disease-associated PAH. PAH patients receiving $\beta$-blockers had a similar survival and time to clinical worsening events compared with patients not receiving them. Functional outcomes were similar, although $\beta$-blocker use was associated with a tendency towards shorter walking distance.

The most recent guidelines in PAH do not recommend the use of $\beta$-blockers, unless recommended by comorbidities, because no convincing data are available on their usefulness and safety $[2,3]$. Although the recent study by BANDYOPADHYAY et al. [1] appears like the last demonstration of $\beta$-blocker inefficiency in the setting of PAH, we rather see it as DE MAN and HANDoko [4] pointed out in their recent editorial, as an emerging signal that the dangers of $\beta$-blockers in $\mathrm{PAH}$ patients are smaller than previously thought. We would also point out that $\beta$-blockers are a wide and heterogeneous group of molecules. $\beta$-blockers differ in terms of $\beta$-adrenergic receptors selectivity, adjunctive effects on $\alpha$-receptors and effects on oxidative stress and inflammation [5]. The "first-generation" compounds, such as propranolol and nadolol, are non-selective agents with equal affinities for blocking $\beta 1$ and $\beta 2$ receptors and no important pharmacological properties other than $\beta$-adrenergic blockade. In addition, the $\beta 2$-subtype is the predominant adrenergic receptor present in the pulmonary vasculature. Blockade of the $\beta 2$-receptors may lead to smooth muscle contraction, which could result in an increase in pulmonary vascular resistance and right ventricle pressure [6]. The "second-generation" of $\beta$-blockers, such as metoprolol or bisoprolol, are $\beta 1$-selective (cardioselective) and lack $\beta 2$-receptor blockade, therefore reducing some of the peripheral and pulmonary side effects. The "third generation" has vasodilating properties in addition to its $\beta$-blockade action. This specific generation has different pharmacological profiles as nebivolol is highly $\beta 1$-selective with vasodilating activity possibly due to an interaction with the L-arginine/nitric oxide pathway while carvedilol blocks $\beta 1, \beta 2$ and $\alpha 1$ adrenergic receptors, the latter possibly acting as potent vasodilator [7]. Hence, the third generation might have the advantage of right heart afterload reduction to counteract the negative inotropic properties of adrenergic withdrawal, which raise interest for this specific generation of molecules as an add-on to current therapies. However, prospective clinical trials must be performed to clarify whether or not targeting the $\beta$-adrenergic system can be added to the current treatment paradigm of PAH [8].

The initial belief that $\beta$-blockers may be harmful in PAH was strengthened by a small study in 10 patients in the setting of porto-pulmonary hypertension, in whom withdrawal of propranolol (eight out of 10) or atenolol (two out of 10) treatment was associated with an improved 6-min walk distance [9] and further strengthened by a case report in which the addition of a single dose of $25 \mathrm{mg}$ oral metoprolol combined with amiodarone caused a near-fatal acute cardiac decompensation [10]. However, this is not surprising since administration of first-generation compounds, such as propranolol, causes a decrease in contractile state. This, plus a concomitant increase in systemic vascular resistance from $\beta 2$-receptor blockade, leads to a profound decrease in cardiac output, which results in a drug intolerance rate of $20 \%$ in dilated cardiomyopathy [7]. Alternatively, no obvious explanation for the $\beta$-blockers and amiodarone interaction exists. An additive effect in patients could be considered as amiodarone has also $\beta$-adrenergic blockade effect [11].

A recent retrospective study dealing mainly with the second-generation of $\beta$-Bs bisoprolol reported that their use was associated with a reduction in right ventricle (RV) dilatation and an improvement of tricuspid annular plane systolic excursion, which was attributed to a reduction of RV fibrosis [12]. However, in two recent retrospective studies, clinical, functional and hemodynamic outcomes of PAH patients were analysed coincidentally taking $\beta$-blockers, e.g. for treatment of arrhythmias. In these studies, second-generation 